\title{
A New Kind of File Uploading of LIMS Based on VB.NET
}

\author{
CaiRangZhuoMa \\ College of Electrical Engineering, Northwest University for Nationalities \\ Lanzhou, Gansu Province, 730030, China
}

\begin{abstract}
At developing of website always come across some problem of file uploading. There are very complication to use some scripts dealing with this problem. In this paper we introduced a way of uploading file which edited with VB.NET. We emphasized to discuss the theory of uploading file which edited by VB. NET, to achieve the key factor of file uploading based on Web mode, principally to comprehend devising project and assaying theory and editing and debugging procedure at laboratory of transferring data from Browse to Web sever, to pass by elaborating tissue and completed, fully meets with user's actual situation and operating requirements. The devised software has completed software planning of requirements tasks, the system based of customer's requirement, remains to more improve and perfection[1,2]. The system has successfully developed which have valuable experience on the experimental teaching in Colleges and has a good developing prospect.
\end{abstract}

Keywords-browse/sever; Visual Studio .NET; file uploading

\section{Introduction}

In the recent years, Internet/Intranet technique has been applied widely in education, scientific research, enterprise and the government departments, these make transferring files on the network become reality requirements, application program's service mode from the past customers/server to browse/server. Traditional static Web pages can not content with the requirement of application requirements. We can transfer files using FTP Protocol on the network, but it has complicated operating commands and unfriendly interfaces, common users flinching and escaping, so people urgently need a friendly interface, simplify operating transferring files technologies on network. However, file uploading of experimentation message management based on HTTP protocol, a new transferring files mode on network emerge as the times require.

\section{Building the Development Platform}

Laboratory has been a very important part of Colleges and Universities, constructing the first-class teaching and faculties based on first-class experimentation platform. Being a basing laboratory which has a lot of teaching task educated students, accelerated the development of faculties. This system is a practicing teaching system based on B/S structure, it adopted multi-technology for examole: ASP.NET[3,4], ADO.NET, SQL Server2000 Database etc, breaking through traditional filing mode, there are advanced technology, friendly interface, strong interactive ability, simplicity operating and high utility. A practicing teaching system based on B/S structure devised the operating database midst layer which used the Web server and ASP.NET. We can combine Database based on C/S mode and Web technology. So there is Database structure which has three layers structure based on B/S mode.

It's working principle : Adopting Browse for example IE and Netscape in the front, the operation information that users submit required HTTP to Web server IIS, IIS is available Database using ASP.NET and ADO.NET and transferring the operating results in front Browser that using HTML pages mode.

1)Development tool: a set of language including $C \#$ and VB.NET; a set of development tool, including Visual Studio .NET (a. the essential system installed b. enterprise development c. system help); a comprehensive class library; Be used execution object's public language running platform in the framework.

2)Special server: a set of .NET enterprise server, called SQL Server2000 , Exchange Server2000, BiztalkServer2000, and etc.They provided special function on storage in relationship Database. Operating system Win XP professional.

3).NET framework is a important part of Microsoft.NET. It is a environment that can establish, deploy and operate Web service. It is running supporting and management in all application program of .NET.

4)Web service Browser IE6.0

5)Visual Studio .NET is a installed environment requirement.

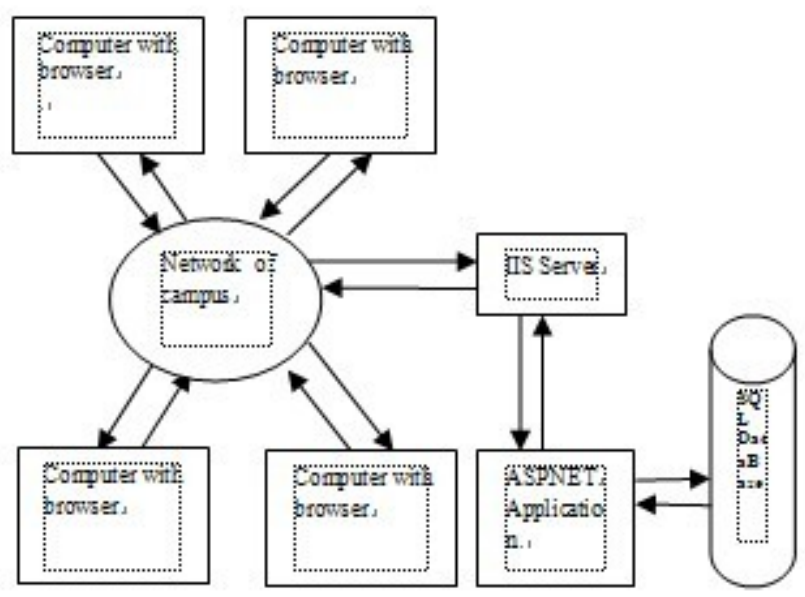

Fig. 1 The method of file uploading using ASP

When we designed the homepages, we can provide downloading service for user using Super Link. But the function of file uploading can not easily use. If we want to 
extend the server's function, commonly used method is CGI. This can complete extending the Web function through storing and fetching server's environment variable and Standard input-output. However, the CGI program very complex when they are edited, a larger workload, specially when we use of CGI to develop VB program, the process more cumbersome.

When we want to create a local Web project under installed VB.NET environment on computer, we must install and configure Internet Information service and frontPage2000 server extensions under local Web project. To install and configure IIS server FrontPage2000 server [5], The following steps:

(1) Opening the Control Panel;

(2) Double-click Add / Remove Programs, and then click " Add / Remove windows components" options, opening the Windows Components Wizard;

(3) Then select IIS options, check IIS;

(4) Clicking "for more information" button, In the pop-up window check Fontpage2000 server extensions options, installation;

(5) At start menu, checking Control Panel, opening manage tools, checking manage computer. In the dialog box, spreading the node of service and application program, and then spreading IIS, Right-click default web site, checking all tasks, in the end checking configured to expand services. If we can't find this, proved Fontpage2000 server expansion has been installed.

\section{System Software Design and Implementation}

\section{A}

\section{ADO.NET Object}

ADO.NET object including: DataSet, DataTable, DataColumn, DataRow and DataRelation.

Using DataRelation object, we must put ForeignKeyConstraint to be added to the revised data sheet of the members of the ConstraintsCollection. ConstraintsCollection the members of the DataTable object determines when a father table's value is deleted of altered, it should perform what action.

After setting up the relations, it will refuse to all changing operation of data sets which have destroy the relationship, and producing a run-time anomaly. When we traversal of the table's records, called the GetChildRows method can access to all relevant lines from connected table. The GetChildRows method returns an array of DataRow objects,this a new record of the hierarchical navigation approach, but traditional way of navigation records belong to the order patterns or random models.

Experimental plan to declare: teachers and management want to exchange depend on experimental declaring. Teachers submitted the declaring plan table to management. The higher authorities can review.

1)Submitted planning declaration: First teachers downloaded planning declaring table and upload after filled it. And then submitted it. when teachers submitted ,it preserved in the directory of jhsb. The file name is system time(date and time) and document size, composed 15 numbers. This can be avoided a document that has a same name with others covered the previous document. Now this ensured the reliability of the system. The submitted plan is a plan which is waiting for declared. It will in the table of waiting for declared.

2) Reviewing plan: Authorities want to see the declaration they must land. All declaring table come from three categories ,they are waiting declaration ,pass and not pass. Administrators have the right to review plans whether it can through.

Clicking declaring table ,We can see the detail of it. Clicking passing review, we can make it through review. Reviewed the plan have been included in the audit program table. Clicking audit does not pass, the plan did not pass examination.

\section{B $\quad$ File Uploading}

Private Sub Button1_Click(ByVal sender As System.Object, ByVal e As System.EventArgs) Handles Button1.Click

Dim mytime As String =

DateTime.Now.ToString("yyyyMMddhhmmss")

Dim nam As String = myFile.PostedFile.FileName

Dim i As Integer = nam.LastIndexOf(".")

Dim newext As String = nam.Substring(i)

'Access to the file extension.

Dim newname As String = mytime +

myFile.PostedFile.ContentLength.ToString ' According to different of the file size and date access to the file name,To ensure that the file name does not repeat.

Label5.Text = "jhsb/" + newname + newext

'Dim exits As Boolean

' determine whether there is folder

mytime)

' exits = System.IO.Directory.Exists("jhsb/" +

' If exits = False Then mytime)

'System.IO.Directory.CreateDirectory("jhsb/" +

'End If

myFile.PostedFile.SaveAs(Server.MapPath("jhsb/" + newname + newext)) ' Saving the file in the directory jhsb.

myFile. Visible $=$ False

Button1. Visible $=$ False

Label4. Visible $=$ True

TextBox1.Enabled $=$ True

End Sub

\section{Conclusion}

In this paper, through researching and development of application Web Database development technology on information manage system, to achieve file uploading based on Web mode. The key is we need understand when the client browser to the Web server submits data, the mode of transfer to web. We take full account of user's actual situation and requirement from designing plan, analyzing theoretical and programming and debugging in laboratory to well-organized and complete. The software that we designed completes the task demands of designed software. The system will further improve and enhance according to user needs. The successful development of the system has 
provided valuable experience on teaching experiment in college [6]. It has good prospects for the development.

\section{References}

[1] Liu Ying, Zhang Shuguang. Based on B / S Experimental Management System Design and

Implementation. Journal of Wuhan University (Engineering), 2002.2.

[2] Xue Cheng. Management Information System. Beijing: Tsinghua University Press, 1993.2.
[3] Chao Huang, Ting-Ting Li. Visual Basic.NET project development practice. China Railway

Press, 2003.10.

[4] Chang Hsin-East. Guide the development of information systems the use of Visual Basic.NET and SQL Server. Beijing: Tsinghua University Press, 2003.3.

[5] Li Ping, Zhang Wen. SQL Server2000 Chinese-based database application. Metallurgical Industry Press, 2002.2.

[6] Zhang Limin. Example learning ASP.NET. Huazhong University of Science and Technology Press, 2003.4. 